\title{
Characterization of gasoline/ethanol blends by infrared and excess infrared
}

\section{spectroscopy}

Stella Corsetti, ${ }^{1,2}$ Florian M. Zehentbauer, ${ }^{1,3}$ David McGloin, ${ }^{2}$ Johannes Kiefer ${ }^{1,3,4 *}$

${ }^{1}$ School of Engineering, University of Aberdeen, Fraser Noble Building, Aberdeen AB24 3UE, Scotland, United Kingdom

${ }^{2}$ SUPA, Division of Physics, School of Engineering, Physics and Mathematics, University of Dundee, Nethergate, Dundee DD1 4HN, Scotland, United Kingdom

${ }^{3}$ Technische Thermodynamik, Universität Bremen, Badgasteiner Str. 1, 28359 Bremen, Germany

4 Erlangen Graduate School in Advanced Optical Technologies (SAOT), Universität Erlangen-Nürnberg, Erlangen, Germany

*corresponding author: Johannes Kiefer

Technische Thermodynamik, Universität Bremen

Badgasteiner Str. 1, 28359 Bremen, Germany

phone: +49421218 64777

e-mail: jkiefer@uni-bremen.de 


\begin{abstract}
Fuels for automotive propulsion are frequently blends of conventional gasoline and ethanol. However, the effects of adding an alcohol to a petrochemical fuel are yet to be fully understood. We report Fourier-transform infrared spectroscopy (FTIR) of ethanol/gasoline mixtures with systematically varied composition. Frequencies shifts and excess infrared absorbance are analyzed in order to investigate the mixture behavior at the molecular level. The spectroscopic data suggest that the hydrogen bonding between ethanol molecules is weakened upon gasoline addition, but the hydrogen bonds do not disappear. This can be explained by a formation of small ethanol clusters that interact via Van der Waals forces with the surrounding gasoline molecules. Furthermore, approaches for measuring the chemical composition of ethanol/gasoline blends by FTIR are discussed. For a simplistic approach based on the Beer-Lambert relation, an optimized set of parameters for quantitative measurements are determined. The best compromise between measurement sensitivity and accuracy is found for the $\mathrm{CO}$ stretching mode of the alcohol. For the traditional method of calibrating the ratio of integrated band intensities of the $\mathrm{CH}$ and $\mathrm{OH}$ stretching regions it is found that narrowing the spectral window of the $\mathrm{CH}$ stretch can significantly improve the measurement sensitivity.
\end{abstract}

Keywords: Biofuel, fossil fuel, alcohol, excess absorption, frequency shift 


\section{Introduction}

The transport sector relies heavily on fossil fuels, for example in the form of gasoline, diesel, compressed natural gas (CNG) and liquefied petroleum gas (LPG). The excessive use of fossil fuels, however, is leading to the depletion of these resources (those that can be accessed easily) and is presumed to contribute to the greenhouse effect, air pollution, acid rain, ozone depletion and climate change. Pollutants generated via combustion of fossil fuels, e.g. carbon monoxide and dioxide, unburned hydrocarbons (UHC), nitrous oxides (NOx), hydrofluorocarbons (HFC), and perfluorocarbons (PFC), pose a serious problem not only for the environment but also for human health. ${ }^{1}$ Hence, there is a strong need for alternative fuels. $^{2}$

Biofuels may provide a feasible solution to these problems due to their natural/renewable origin and their low pollutant emissions when compared to their fossil fuel counterparts. Bio-ethanol is obtained from a variety of feedstocks such as sugar cane, corn, sunflower, fruit, grain, cotton and agricultural waste material such as straw. ${ }^{3}$ It can also be extracted from sugar produced directly from the biosynthesis process of algae, which makes use of sunlight, carbon dioxide and sea water. There are some difficulties, however, in using bio-ethanol directly as an engine fuel. The high viscosity of ethanol hinders the atomization process during fuel injection and contributes to incomplete combustion reducing the engine efficiency and lifetime. ${ }^{4}$

At present, many commercial fuels are mixtures of conventional petrochemical fuels and a certain amount of biofuel. Ethanol/gasoline blends up to $20 \%$ can be used in constant speed engines without any modification and no significant power reduction. ${ }^{5}$ It was observed that a mixture of bio-ethanol with gasoline leads to a reduction of up to $6 \%$ in $\mathrm{CO}$ emission and up to $24 \%$ in NOx emissions when compared to the use of fossil fuel alone. ${ }^{6}$ Blending bio-ethanol with gasoline, however, can significantly alter the fuel properties and thus the 
evaporation and combustion characteristics of the fuel. ${ }^{7}$ As a consequence, the past 20 years have seen significant research effort aimed at understanding the influence of ethanol on the physicochemical properties of fuel blends, for example the vapor pressure ${ }^{8}$, the viscosity ${ }^{9}$, and the density ${ }^{10}$. Other works studied the effects of ethanol addition on the fuel performance in terms of spray formation ${ }^{11,12}$ and combustion behavior ${ }^{13,14}$. Understanding the fundamental nature of the changed properties and behavior, however, is highly desirable as it would help to predict the macroscopic phenomena without extensive experimental testing.

The aim of this work is to develop an understanding of the behavior of ethanol/gasoline mixtures at the molecular level. For this purpose, the ethanol blends of a gasoline surrogate with systematically varied ethanol content are studied using Fourier-transform infrared spectroscopy (FTIR). In the past, IR and near-IR spectroscopy was mainly used for determining the ethanol content in gasoline in a small number of studies. ${ }^{15-17}$ In this paper, we analyze the IR spectra of ethanol/gasoline blends across the full composition range, i.e. from pure ethanol to pure gasoline surrogate in systematically varied concentration steps. Frequency shifts as well as excess infrared absorbance are evaluated in order to infer information about molecular interactions in the mixtures. Furthermore, methods for determining the chemical composition of the mixtures from the IR spectra are discussed.

\section{Experimental Section}

Fuel blends. A gasoline surrogate was prepared by mixing iso-octane (2,2,4-trimethylpentane, purity $>99 \%$ ) and n-heptane (purity $>95 \%$ ) with a ratio of 1:1 by weight. Different ethanol/gasoline blends were prepared with systematically varied ethanol (purity > 99\%) percentage (by weight) in $10 \%$ increments. The samples span the entire composition range 
from pure gasoline to pure ethanol. All blends were prepared gravimetrically using an analytical balance.

FTIR spectroscopy. The IR spectra of the solutions were collected over the range from 500 to $4000 \mathrm{~cm}^{-1}$ using a Bruker Vertex v70 spectrometer. The nominal resolution was $1 \mathrm{~cm}^{-1}$. In order to increase the signal to noise ratio, for every sample 32 scans were averaged. The instrument was equipped with an attenuated total reflection (ATR) module (diamond, one reflection, $45^{\circ}$ ). During the measurements the samples on the ATR crystal were covered with a small glass cap to avoid sample evaporation. All measurements were carried out at $294 \mathrm{~K}$.

\section{Results and Discussion}

The IR spectra are analyzed in different ways reflected by the organization of this section. In a first step, the unprocessed spectra are studied with respect to peak positions and frequency shifts. Thereafter, the excess absorbance spectra are calculated and analyzed. Eventually, methods for obtaining compositional analysis are tested.

\subsection{Infrared spectra}

The infrared spectra of the gasoline surrogate, the pure ethanol and the blends are displayed in Fig.1. There are no absorption peaks from gasoline in the $\mathrm{OH}$ stretching region between 3000 and $3600 \mathrm{~cm}^{-1}$ and in the $\mathrm{CO}$ stretching region from 1020 to $1120 \mathrm{~cm}^{-1}$, while ethanol exhibits distinct bands in those regions. The peak absorbance of the $\mathrm{OH}$ stretching band is observed at $3319 \mathrm{~cm}^{-1}$. Further characteristic features of ethanol can be found in the fingerprint region. The dominant feature is a peak doublet at 1046 and $1088 \mathrm{~cm}^{-1}$, which can be assigned to the 
symmetric and asymmetric $\mathrm{CO}$ stretches, respectively. In the $\mathrm{CH}$ bending and stretching regions (1200-1600 $\mathrm{cm}^{-1}$ and $2800-3000 \mathrm{~cm}^{-1}$, respectively) overlapping vibrational bands of ethanol and gasoline can be found.

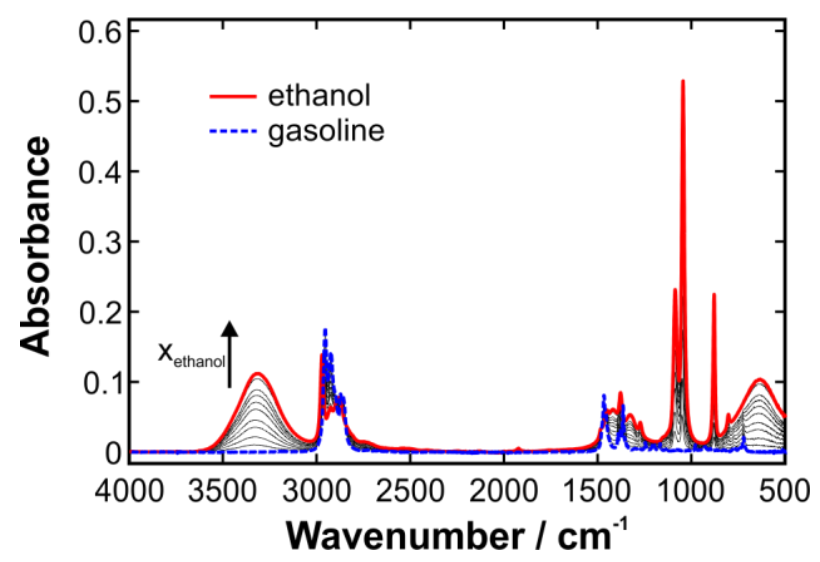

Figure 1: FTIR spectra of the gasoline surrogate, the pure ethanol and the blends.

Figure 2 shows the enlarged $\mathrm{CH}$ stretching region where contributions from ethanol and gasoline alkyl groups overlap. However, there are sub-regions, which are dominated by either the gasoline or the ethanol. Ethanol vibrational modes are identified at 2973, 2928 and 2881 $\mathrm{cm}^{-1}$. The predominant bands of the hydrocarbons in the gasoline surrogate can be found at 2956 and $2925 \mathrm{~cm}^{-1}$ (asymmetric $\mathrm{CH}$ stretching vibrations) as well as 2873 and $2861 \mathrm{~cm}^{-1}$ (symmetric $\mathrm{CH}$ stretching vibrations). The gasoline spectrum crosses the ethanol spectrum in the isosbestic points at 2966, 2896, 2876, and $2846 \mathrm{~cm}^{-1}$. These points represent the wavenumbers at which the absorbance of the mixture remains invariant and ethanol and gasoline absorb the same amount of light. The range between 2966 and $2896 \mathrm{~cm}^{-1}$ (highlighted in Fig. 2) is dominated by gasoline but with a background from ethanol. Considering the entire $\mathrm{CH}$ stretching region, the integrated absorbance decreases with increasing percentage of ethanol. 


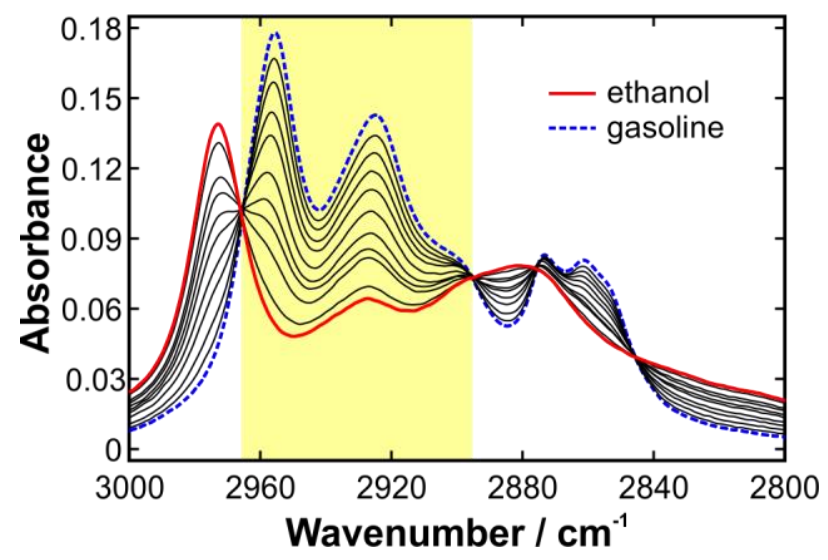

Figure 2: FTIR spectra in the CH stretching region. The wavenumber range between the two isosbestic points at 2966 and $2896 \mathrm{~cm}^{-1}$ is highlighted.

The $\mathrm{CH}$ bending region is displayed enlarged in Fig. 3. As found in the stretching region, contributions from ethanol and gasoline methyl and methylene groups can be observed. Again, there are sub-regions, which are dominated either by the gasoline or by the ethanol bands. Peaks from ethanol appear at 1455, 1448, 1419, 1479, 1328 and $1275 \mathrm{~cm}^{-1}$, peaks from the gasoline components at $1468,1393,1379,1365,1353,1282,1248,1207 \mathrm{~cm}^{-1}$. Isosbestic points can be found at 1480, 1459, 1368 and $1369 \mathrm{~cm}^{-1}$. The range between 1480 and 1459 $\mathrm{cm}^{-1}$ (highlighted in Fig. 3) is dominated by gasoline but with a background from ethanol. 


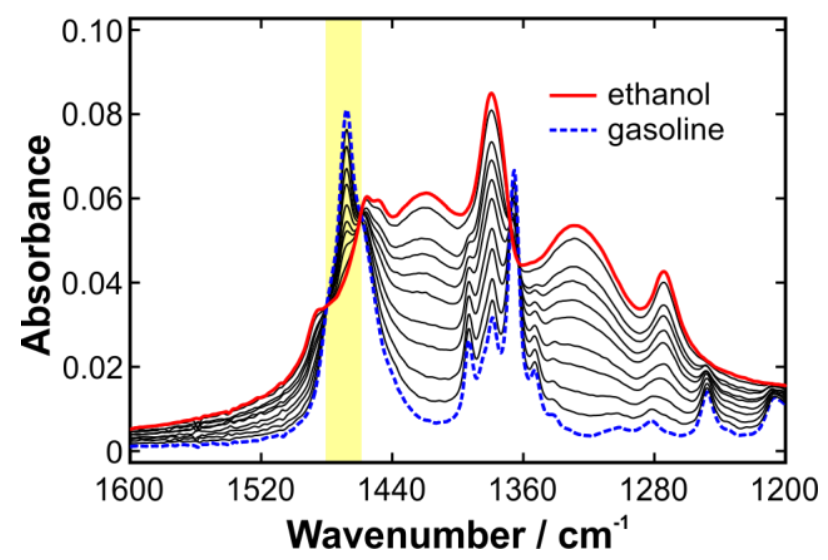

Figure 3: FTIR spectra in the CH bending region. The wavenumber range between the two isosbestic points at 1480 and $1459 \mathrm{~cm}^{-1}$ is highlighted.

Figure 4 shows the asymmetric and symmetric CO stretching modes of ethanol. They represent rather isolated lines at 1088 and $1046 \mathrm{~cm}^{-1}$, respectively. Interestingly, both peaks are characterized by distinct and systematic frequency shifts to higher wavenumber with respect to their position in pure ethanol dependent on blend composition. Such a blue shift indicates a strengthening of the $\mathrm{CO}$ bond, which is most likely caused by a weakening of the intermolecular hydrogen bonds between ethanol molecules.

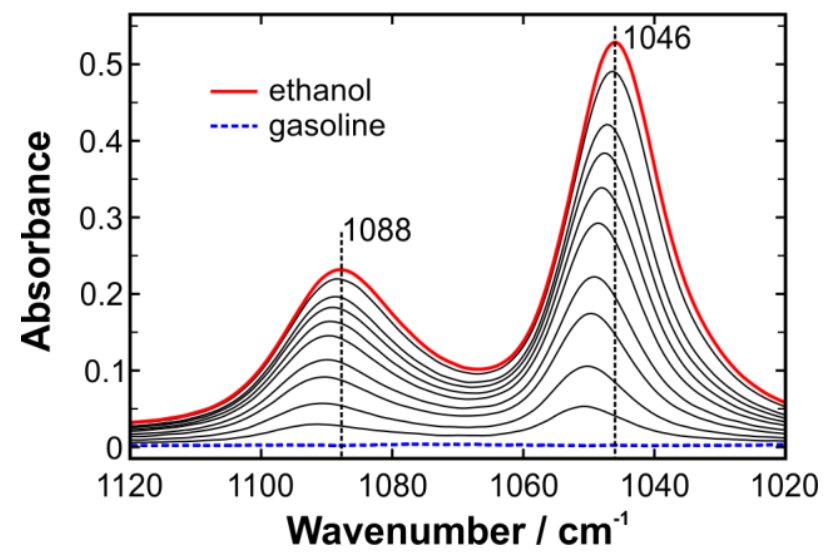


Figure 4: FTIR spectra in the CO stretching region. The vertical dashed lines indicate the wavenumbers of the peaks in pure ethanol.

In order to obtain a clearer view of this phenomenon, Fig. 5 shows the frequency shifts for three individual ethanol peaks as function of ethanol mole fraction. The plotted wavenumber shifts represent the difference of peak wavenumber in a blend spectrum and the peak wavenumber of the corresponding peak in the spectrum of pure ethanol. Frequency shifts were observed for the CO stretching modes $\left(1088\right.$ and $\left.1046 \mathrm{~cm}^{-1}\right)$ and the CC stretching mode $\left(880 \mathrm{~cm}^{-1}\right)$ of ethanol. Upon adding gasoline all three vibrational bands are blue-shifted (for completeness we note that the $\mathrm{OH}$ stretching band also shows a blue shift, but the magnitude of the shift is very small compared to the spectral width of the band). Higher vibrational frequency indicates a stronger covalent bond. This effect is due to weakening of the intermolecular interactions between the ethanol molecules. Van der Waals forces are dominant in the mixtures with high gasoline content while in ethanol hydrogen bonding is the predominant interaction mechanism. The characteristic bands of the gasoline did not show a detectable frequency shift. At the macroscopic level, the strength of intermolecular interactions influences the vapor pressure and hence the evaporation of the blends. For instance, weaker intermolecular interactions generally indicate a faster evaporation rate in a fuel spray. 


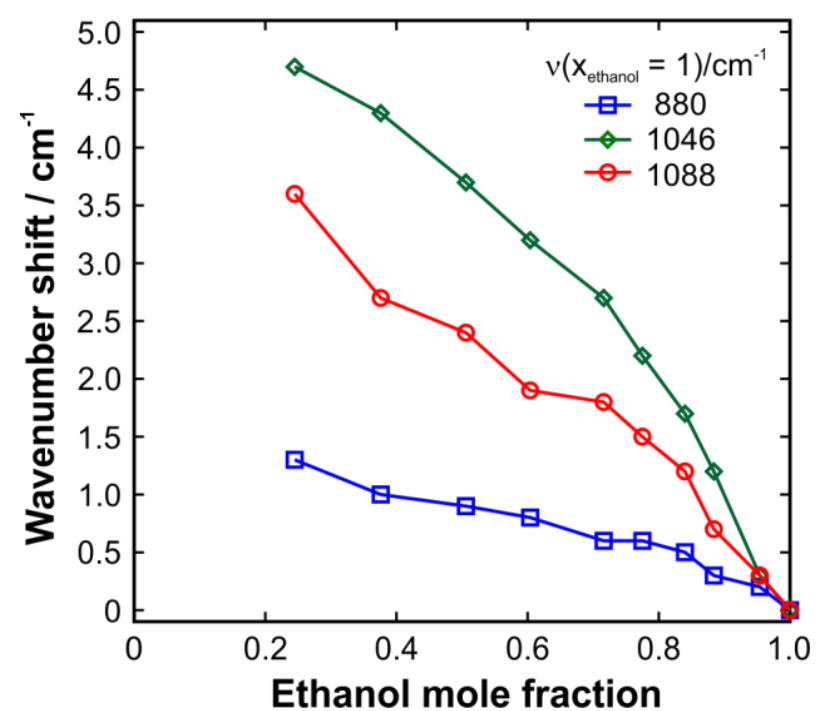

Figure 5: Frequency shifts of the CC $\left(880 \mathrm{~cm}^{-1}\right)$ and CO stretching $\left(1046 \mathrm{~cm}^{-1}\right.$ symmetric and $1088 \mathrm{~cm}^{-1}$ asymmetric) modes of ethanol as function of blend composition.

\subsection{Excess absorbance spectra}

In recent years, excess infrared absorption spectroscopy has found a number of applications for studying solvent mixtures. ${ }^{18-20}$ It can be used to enhance the spectral resolution of the IR bands under study and look at changes in the dipole moment, reflecting changes in the charge distribution of the vibrating system. The procedure of calculating excess IR spectra was introduced by Li et al. ${ }^{18}$ lending the concept of thermodynamic excess properties. To do so, the ideal IR absorbance of a mixture can be calculated as the sum of the absorbances of the pure components weighted by their molar fraction. In the present case, the ideal absorbance $A^{\text {ideal }}$ of a gasoline/ethanol blend is given by

$$
A^{\text {ideal }}=X_{\text {gasoline }} A_{\text {gasoline }}^{0}+X_{\text {ethanol }} A_{\text {ethanol }}^{0}
$$


where is $x_{i}$ and $A^{0}{ }_{i}$ are the mole fraction and the absorbance of the pure component $\mathrm{i}$, respectively. The excess spectrum is defined as the difference between the spectrum of a real mixture (experimental spectrum) and the spectrum of the corresponding ideal one. Hence, the excess absorbance is given by

$$
A^{\text {excess }}=A_{\text {mixture }}^{\text {real }}-A_{\text {mixture }}^{\text {ideal }}
$$

Depending on the sign of this absorbance, different conclusions on the IR activity of the real mixture with respect to that of an ideal one can be made. Positive excess absorbance values mean higher IR activity in the real solution, negative values mean a smaller one.

Figure 6 shows the excess absorbance spectra of the gasoline surrogate, the pure ethanol and the blends. By definition, the excess absorbance of the pure substances is zero. In the blends, the excess absorbance is mainly negative across the spectrum (panel A of Fig. 6). This means that the real mixture appears less IR active than an ideal one would be. However, in the $\mathrm{CH}$ stretching region (Fig. 6B) the excess absorbance has significant positive features. We note that it is positive exactly in the range between the isosbestic points identified in the IR spectra, which is dominated by gasoline (highlighted range in Fig. 2). A positive excess absorbance indicates that the $\mathrm{CH}$ bonds exhibit greater IR activity in the real mixture than they would have in an ideal solution. This indicates an enhanced dipole moment. 

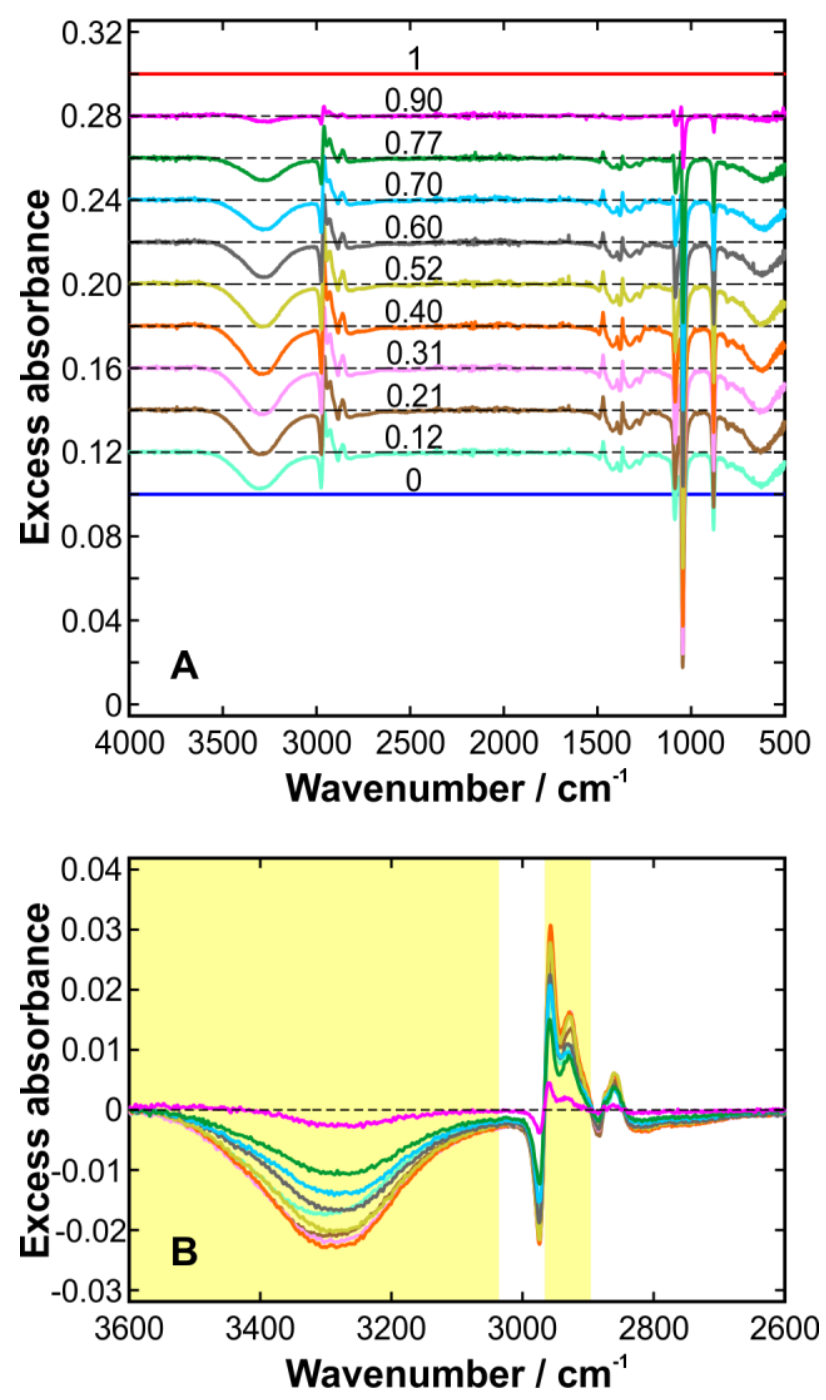

Figure 6: Excess absorbance spectra of the gasoline surrogate, the pure ethanol and the blends. Panel A: full spectrum; the spectra of the individual blends are plotted with 0.02 incremental offset; the dashed lines indicate the baseline for each individual curve. Panel B: $\mathrm{OH}$ and $\mathrm{CH}$ stretching region; the ranged over which the excess absorbance was integrated for Figure 7 are highlighted.

Figure 7 shows the excess absorbance integrated over characteristic spectral bands and normalized with respect to the width of integration window. As function of mixture composition rather symmetric distributions of the integrated excess absorbance can be 
observed. The $\mathrm{CH}$ stretching (2966-2896 cm $\left.\mathrm{cm}^{-1}\right)$ and $\mathrm{CH}$ bending $\left(1480-1459 \mathrm{~cm}^{-1}\right)$ sub-regions dominated by gasoline show positive excess absorbance. The $\mathrm{OH}\left(3614-3036 \mathrm{~cm}^{-1}\right), \mathrm{CO}$ (1107-1011 $\left.\mathrm{cm}^{-1}\right)$ and CC (895-866 $\left.\mathrm{cm}^{-1}\right)$ stretching bands characteristic for ethanol show negative excess absorbance. Positive values indicate that the infrared activity is enhanced compared to the pure components, which indicates a stronger dipole moment; negative values vice versa. Interestingly, the $\mathrm{OH}$ band shows the smallest deviation of the ethanol bands, although it is directly involved in the hydrogen bonding interactions. Hence, intuitively a strong change is expected when the hydrogen bond is replaced or sufficiently weakened so that a frequency shift is observed. The observed behavior suggests that the ethanol molecules form small clusters in which they still interact through hydrogen bonding but in a weaker network than in pure ethanol, and the interaction with the surrounding gasoline phase is through van der Waals forces at the ethyl moiety. The weakening of the hydrogen bonding network plus enhanced van der Waals forces at the aliphatic chain can also explain the observed frequency shifts in Fig. 5. Such self-association effects of aliphatic alcohols in hydrocarbons have been reported in the literature many times. ${ }^{21,22}$

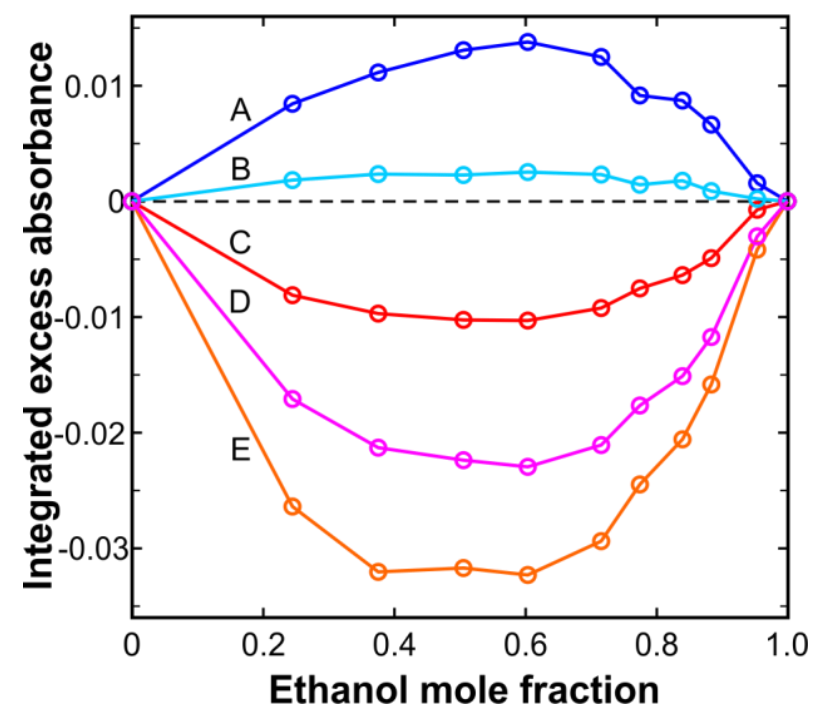


Figure 7: Excess absorbance integrated over characteristic spectral ranges as function of blend composition: the gasoline $C H$ stretching band $(A)$, the gasoline $C H$ bending band $(B)$, the ethanol $\mathrm{OH}$ stretching band $(C)$, the ethanol CO stretching band $(D)$, and the ethanol CC stretching band (E). For details see text.

\subsection{Quantitative measurements}

Vibrational spectroscopy is frequently used for compositional analysis of mixtures. There are many different approaches for extracting a quantitative piece of information from a spectrum. For liquid and gaseous hydrocarbon mixtures, spectral soft modeling has proved its potential. ${ }^{23,24}$ However, a pre-requisite for applying soft modeling is that there are no peak shifts or non-idealities in the line shapes and intensities. In other words, the excess spectrum must be essentially zero for all mixtures. Hence, soft modeling is not the method of choice in the present gasoline/ethanol system. An alternative approach would be indirect spectral hard modeling. ${ }^{25}$ The hard modeling can handle peak shifts and other non-idealities, but the effort is significantly higher and may not be justified in a relatively simple (chemically speaking) system like the one discussed here. The same is true for chemometric methods, which often require large data sets for calibration and training of the algorithms. ${ }^{17,26}$

Straightforward approaches for quantifying IR spectra make use of the Beer-Lambert relation, which states a linear relationship between absorbance and the concentration of a target substance. For example, Battiste et al. ${ }^{16}$ utilized the ethanol peaks at 880 and $1050 \mathrm{~cm}^{-1}$ to determine the ethanol content in gasoline/alcohol blends. Balabin et al. ${ }^{15}$ used the $\mathrm{OH}$ stretching band for this purpose. In order to find the optimum feature for signal quantification in the spectrum, we use a method developed for analyzing carbohydrates and catalyst molecules dissolved in ionic liquids. ${ }^{27-29}$ For each spectral data point (7259 in our case), the 
absorbance values of the IR spectra are plotted against the ethanol mole fraction and then fitted with a linear function, empirically representing the Beer-Lambert relation. The slope of the fitted function indicates how sensitive changes in the ethanol concentration are reflected by changes in the absorbance. Hence, a high absolute value of the slope is desirable. The other crucial parameter is the goodness of the fit given by the coefficient of determination $R^{2}{ }^{29}$ The value of $\mathrm{R}^{2}$ is close to 1 when the data points show a good correlation.

Figure 8 displays the spectra of the slope and $\mathrm{R}^{2}$ values. In the regions where little or no signal is present (see Fig. 1), the slope is essentially zero and the $\mathrm{R}^{2}$ is low or very noisy. In the regions where ethanol is predominantly absorbing, the slope is positive, and negative slope indicates regions characteristic of the gasoline. At the isosbestic points, the slope is zero and the $\mathrm{R}^{2}$ value is very low. All positions where significant local extreme values in the slope spectrum are found are summarized in Table 1 together with the corresponding $\mathrm{R}^{2}$ values. The $\mathrm{R}^{2}$ values listed are above 0.92 , but they do not exceed 0.98 . The latter may be desired for measuring the ethanol content with good accuracy. Good compromises can often be found slightly off-peak. For example, the largest slope and thus the best sensitivity is found at 1046 $\mathrm{cm}^{-1}$. However, the $\mathrm{R}^{2}$ at the peak is only 0.935 and therefore the measurement accuracy is limited. A good compromise is found at $1050 \mathrm{~cm}^{-1}$, where the $\mathrm{R}^{2}$ value is 0.980 and the slope is only reduced by $12 \%$ (compared to the maximum). The optimal spectral positions are summarized in Table 2 . The criterion was $\mathrm{R}^{2} \geq 0.98$ or the presence of a local $\mathrm{R}^{2}$ maximum. 


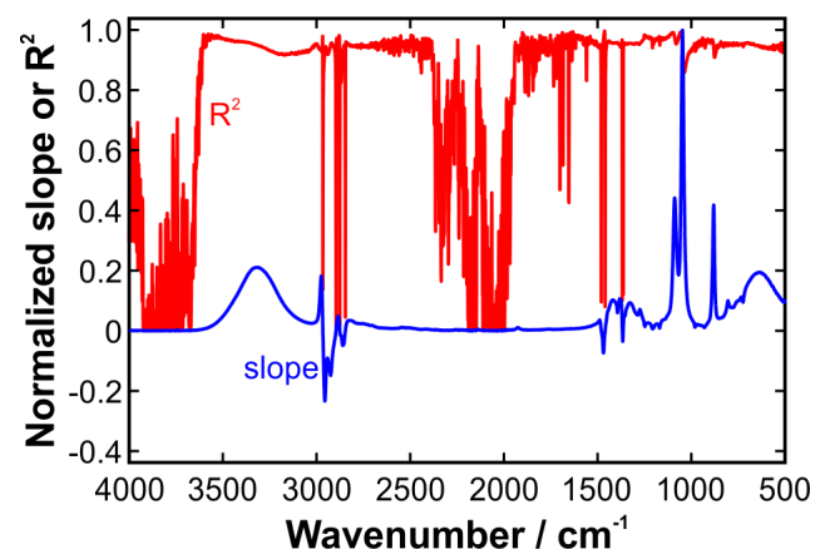

Figure 8: Slope spectrum and fit goodness of the best-fit linear functions. The slope values are normalized to their maximum value.

Table 1: Selected local extreme values of the slope spectrum.

\begin{tabular}{|c|c|c|}
\hline Wavenumber $/ \mathrm{cm}^{-1}$ & $\begin{array}{c}\text { Normalized slope /arbitr. } \\
\text { Unit }\end{array}$ & $\mathrm{R}^{2}$ \\
\hline 3309 & 0.211 & 0.946 \\
\hline 2974 & 0.182 & 0.930 \\
\hline 2955 & -0.234 & 0.934 \\
\hline 2924 & -0.149 & 0.951 \\
\hline 1468 & -0.074 & 0.970 \\
\hline 1366 & -0.033 & 0.926 \\
\hline 1088 & 0.442 & 0.976 \\
\hline 1046 & 1.000 & 0.935 \\
\hline
\end{tabular}




\begin{tabular}{|c|c|c|}
\hline 880 & 0.418 & 0.940 \\
\hline 633 & 0.194 & 0.944 \\
\hline
\end{tabular}

Table 2: Optimum spectral positions for accurate measurement; ${ }^{a}$ reduction in slope compared to peak value, ${ }^{b}$ local maximum of $R^{2}$.

\begin{tabular}{|c|c|c|}
\hline Wavenumber $/ \mathrm{cm}^{-1}$ & $\begin{array}{c}\text { Normalized slope /arbitr. } \\
\text { Unit }\end{array}$ & $\mathrm{R}^{2}$ \\
\hline 3521 & $0.034(84 \%)^{\mathrm{a}}$ & 0.980 \\
\hline 2967 & $0.041(77 \%)^{\mathrm{a}}$ & 0.980 \\
\hline $2951^{b}$ & $-0.194(17 \%)^{\mathrm{a}}$ & 0.945 \\
\hline $2920^{b}$ & $-0.134(10 \%)^{\mathrm{a}}$ & 0.957 \\
\hline 1466 & $-0.059(20 \%)^{\mathrm{a}}$ & 0.982 \\
\hline $1462^{\mathrm{b}}$ & $-0.022(70 \%)^{\mathrm{a}}$ & 0.996 \\
\hline $1364^{b}$ & $-0.026(21 \%)^{\mathrm{a}}$ & 0.953 \\
\hline 1090 & $0.434(2 \%)^{\mathrm{a}}$ & 0.981 \\
\hline $1098^{\mathrm{b}}$ & $0.245(45 \%)^{\mathrm{a}}$ & 0.992 \\
\hline 1050 & $0.884(12 \%)^{\mathrm{a}}$ & 0.980 \\
\hline $1055^{\mathrm{b}}$ & $0.457(54 \%)^{\mathrm{a}}$ & 0.993 \\
\hline
\end{tabular}




\begin{tabular}{|c|c|c|}
\hline $886^{\mathrm{b}}$ & $0.230(45 \%)^{\mathrm{a}}$ & 0.967 \\
\hline $697^{\mathrm{b}}$ & $0.157(19 \%)^{\mathrm{a}}$ & 0.958 \\
\hline
\end{tabular}

Another very common and robust method is to calibrate the intensity (absorbance) ratio of two or more characteristic bands against the mixture composition. ${ }^{23,30,31}$ The traditional approach in the gasoline/ethanol system would be to determine the integrated absorbance of the $\mathrm{CH}$ stretching and the $\mathrm{OH}$ stretching bands and plot their ratio as function of ethanol content (e.g. in terms of mass fraction). In the present work, the integration of the bands was done by summing up the absorbance values of the individual data points inside the set spectral window. The calibration data are shown as squares in Fig. 9. It can be seen that the resulting data points exhibit a linear relationship; the solid line represents the best-fit linear function. This offers a straightforward means of quantification of the IR signals. However, in Fig. 2 it was found that there are spectral sub-regions in the $\mathrm{CH}$ stretching band which are dominated by the gasoline modes and others are characteristic for ethanol. As a consequence, integrating the entire $\mathrm{CH}$ band may result in a loss of sensitivity when using the intensity ratio approach. The circles in Fig. 9 represent the intensity ratio when only the wavenumber range between the two isosbestic points at 2966 and $2896 \mathrm{~cm}^{-1}$ is integrated. The data points do not exhibit a linear relationship any longer, but can be fitted with high goodness using a second order polynomial function (solid line). The slope of the fitted function is larger than the one determined in the previous case. This holds for all data points across the full concentration range. Hence, narrowing the integration window for the $\mathrm{CH}$ band helps to improve the sensitivity of the measurement. However, if raw data with low signal to noise ratio need to be evaluated, limiting the integration window may result in a significant reduction of the 
measurement precision; thus, a lower sensitivity may be justified in the interest of improved precision.

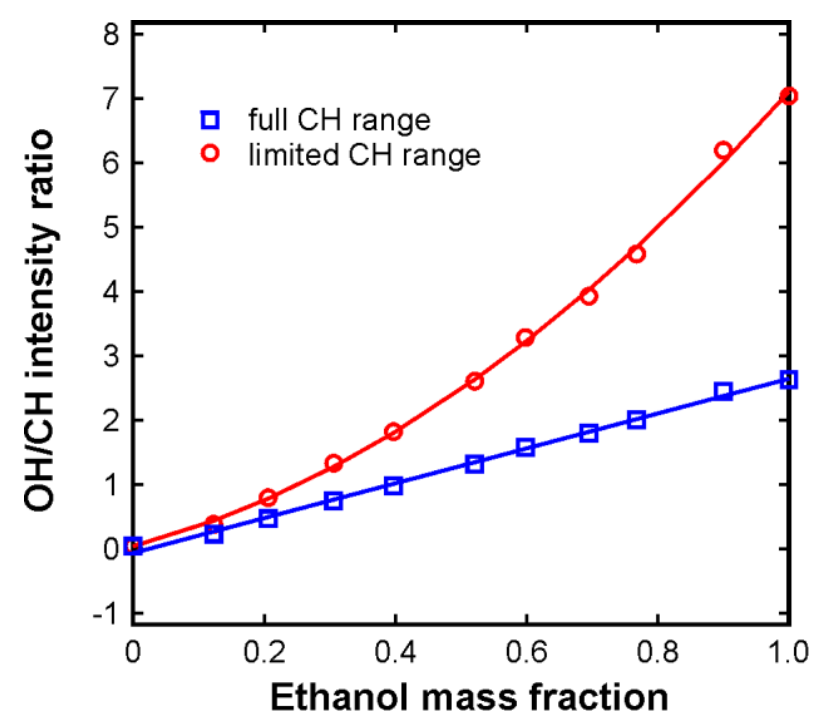

Figure 9: Calibration curves for the intensity ratio of the $\mathrm{OH}$ and $\mathrm{CH}$ stretching bands. For the squares, the full $\mathrm{CH}$ stretching range and for the circles the limited range characteristic for the gasoline was used. The solid lines represent linear and quadratic best-fit functions.

In order to evaluate the performance of the three approaches (the $\mathrm{OH} / \mathrm{CH}$ intensity ratio utilizing the full and limited $\mathrm{CH}$ ranges, and the Beer-Lambert method utilizing the signal of a single data point at $1055 \mathrm{~cm}^{-1}$ ), a leave-one-out cross validation as well as a statistical assessment was carried out. The leave-one-out cross validation is a common tool to evaluate the calibration robustness. For this purpose, one data point is removed from the calibration data set. The calibration function is then determined from the remaining data points. Eventually, the signal intensity of the removed data point is fed into the calibration function as a blind value in order to determine the composition. This procedure was repeated with all individual data points. The residuals, i.e. the deviation of the mass fraction value determined 
from the calibration from the gravimetrically set value, are plotted in Fig. 10. At ethanol mass fraction above 0.3 , the residuals are all within the $+/-5 \%$ corridor. At lower ethanol concentration, the relative deviations are larger, in particular for the Beer-Lambert method because a single data point in the spectrum was used. The highest deviation is found for pure gasoline. This is reasonable as in this case the spectrum of pure gasoline is removed for the calibration and hence the determined calibration curve must be extrapolated, while for ethanol mass/molar fractions $0<w, x<1$ the curve can be interpolated. Moreover, the intensity of the $\mathrm{OH}$ band approaches zero for pure gasoline, which leads to higher uncertainty due to noise. On the other hand, the deviation at the low gasoline end is relatively small. This can be explained by the fact that both $\mathrm{CH}$ ad $\mathrm{OH}$ bands are finite for pure ethanol. Consequently, noise and small errors have less influence on the uncertainty.

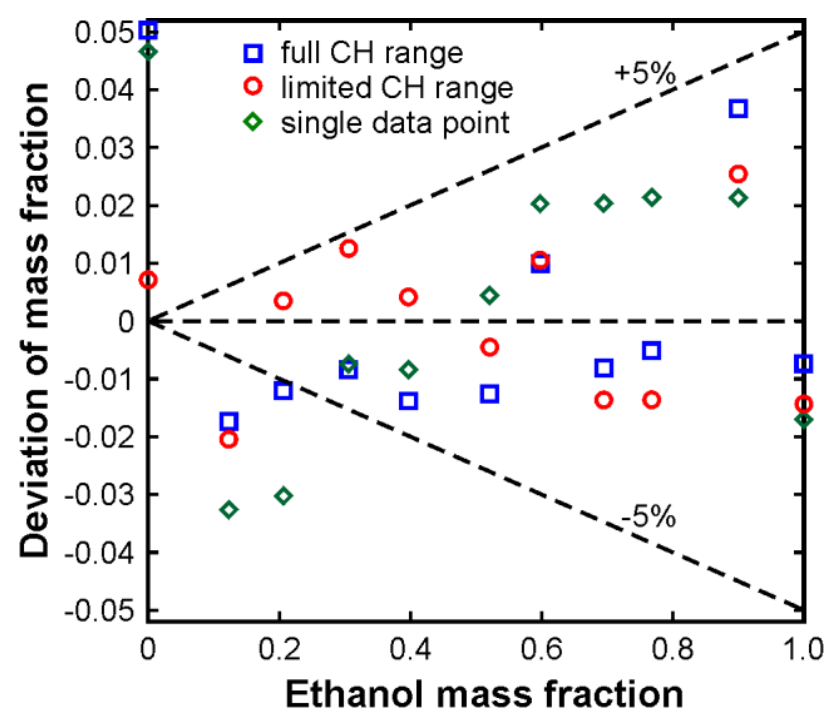

Figure 10: Residuals from the leave-one-out cross validation. The dashed lines indicate the corridor of $+/-5 \%$ deviation from the absolute value. 
The precision of the three methods is determined by the level of noise in the spectra. In order to do a systematic assessment, we simulated signals with different noise levels (random normal distribution) and fed them into the evaluation routines. For each case, one hundred signals were considered. The resulting precision, i.e. the standard deviation of the concentration result divided by the corresponding mean value, is plotted as a function of the noise level in Fig. 11. The symbols represent the data determined from the simulated signals while the solid lines are propagation of error calculations. It can be clearly seen that the standard deviation of the Beer-Lambert approach is about three orders of magnitude higher than the methods utilizing intensity ratios. As we utilize a single data point in the spectrum for the Beer-Lambert analysis, the noise level directly translates into measurement uncertainty. In the other cases, the intensity ratio is determined from integration over certain spectral windows and, consequently, the random noise averages out. Thus, even at high noise levels the measurement precision is reasonably good. It should be noted that the precision of the Beer-Lambert approach can, in principle, be improved by integrating over a certain spectral range.

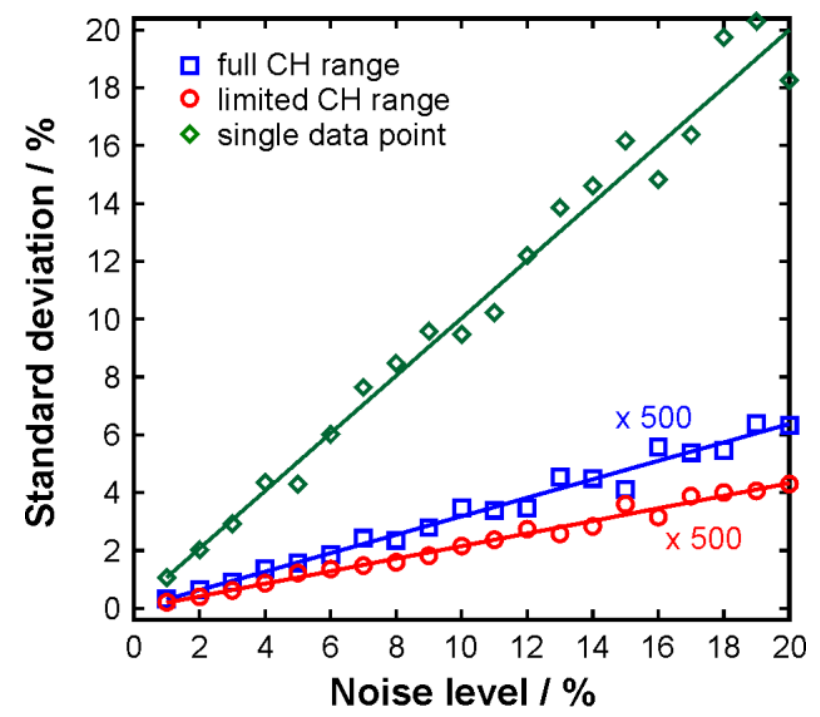


Figure 11: Relative standard deviation of the three evaluation methods as a function of the noise level in the spectra. The symbols show the values determined from 100 simulated signals and the solid lines indicate the trend determined from the propagation of error.

\section{Summary and Conclusion}

In this paper we used Fourier transform infrared (FTIR) spectroscopy for characterizing blends of a gasoline surrogate (a binary mixture of n-heptane and iso-octane) and ethanol. The first part of the work was concerned with phenomena at the molecular level. Systematic shifts towards higher frequency were observed for the alcoholic $\mathrm{CC}$ and $\mathrm{CO}$ (and $\mathrm{OH}$ ) stretching modes. This indicates that the hydrogen bonding network of ethanol is weakened upon addition of gasoline. However, the magnitude of the frequency shifts suggests that the hydrogen bonds do not completely disappear, which can be explained by the formation of small ethanol clusters that interact via Van der Waals forces with the surrounding gasoline molecules. These findings were supported by the results of the analysis of the excess infrared spectra.

In the second part of the paper, approaches for measuring the chemical composition of gasoline/ethanol blends were discussed. Two straightforward methods were identified allowing the quantification of IR spectra with reasonable calibration and computational effort. The first approach used a simple algorithm to identify features in the spectrum that allow quantification via the common Beer-Lambert relation. A systematic analysis helped to find those positions in the spectrum where either the sensitivity to concentration changes is highest, or the measurement accuracy is best, or a good compromise between the two was found. The second approach is based on calibration of the intensity ratio of two characteristic 
bands $(\mathrm{CH}$ and $\mathrm{OH}$ stretching modes). It was found that limiting the spectral window for integrating the band intensities leads to an improved sensitivity, but also to nonlinear behavior. The robustness and measurement uncertainty of the methods was evaluated using a leave-one-out cross validation and a statistical analysis, respectively. The intensity ratio approach was found to provide good precision even at high levels of noise in the spectroscopic data.

In conclusion, IR spectroscopy is a suitable and useful tool for characterizing gasoline/ethanol blends. It does not only help to understand the phenomena at the molecular level, but also provides a fast and straightforward means for measuring the chemical composition of a blend with high accuracy and precision.

\section{Acknowledgement}

This work was supported by the Northern Research Partnership (NRP) in Scotland and the Scottish Sensor Systems Centre (SSSC) funded by the Scottish Funding Council (SFC).

\section{References}

(1) A. Demirbas Progress in Energy and Combustion Science 2007; 33, 1.

(2) Sangeeta, S. Moka, M. Pande, M. Rani, R. Gakhar, M. Sharma, J. Rani, A. N. Bhaskarwar Renewable and Sustainable Energy Reviews 2014; 32, 697.

(3) A. K. Agarwal Progress in Energy and Combustion Science 2007; 33, 233.

(4) J. Xue, T. E. Grift, A. C. Hansen Renewable and Sustainable Energy Reviews 2011; 15, 1098.

(5) A. C. Hansen, Q. Zhang, P. W. L. Lyne Bioresource Technology 2005; 96, 277. 
(6) E. A. Ajav, B. Singh, T. K. Bhattacharya Biomass and Bioenergy 1999; 17, 357.

(7) Z. J. Predojevic, B. D. Skrbic, N. L. Durisic-Mladenovic Fresenius Environmental Bulletin 2011; 20, 1401.

(8) J. A. Pumphrey, J. I. Brand, W. A. Scheller Fuel 2000; 79, 1405.

(9) K. Y. Lee Journal of Hazardous Materials 2008; 160, 94.

(10) L. M. Rodriguez-Anton, M. Hernandez-Campos, F. Sanz-Perez Fuel 2013; 112, 178.

(11) T. Knorsch, M. Heldmann, L. Zigan, M. Wensing, A. Leipertz Experiments in Fluids 2013; 54, 1522.

(12) T. N. C. Anand, A. Mohan, Madan, R. V. Ravikrishna Fuel 2012; 102, 613.

(13) L. Chen, R. Stone, D. Richardson Proceedings of the Institution of Mechanical Engineers Part D-Journal of Automobile Engineering 2012; 226, 1419.

(14) R. Lemaire, E. Therssen, P. Desgroux Fuel 2010; 89, 3952.

(15) R. M. Balabin, R. Z. Syunyaev, S. A. Karpov Energy and Fuels 2007; 21, 2460.

(16) D. R. Battiste, S. E. Fry, F. T. White, M. W. Scoggins, T. B. McWilliams Analytical Chemistry 1981; 53, 1096.

(17) L. S. Mendes, F. C. C. Oliveira, P. A. Z. Suarez, J. C. Rubim Analytica Chimica Acta 2003; 493, 219.

(18) Q. Li, N. Wang, Q. Zhou, S. Sun, Z. Yu Applied Spectroscopy 2008; 62, 166.

(19) J. Kiefer, M. Molina Martinez, K. Noack ChemPhysChem 2012; 13, 1213.

(20) A. Ellis, F. M. Zehentbauer, J. Kiefer Physical Chemistry and Chemical Physics 2013; 15,1093

(21) H. C. Van Ness, J. Van Winkle, H. H. Richtol, H. B. Hollinger Journal of Physical Chemistry 1967; 71, 1483.

(22) J. T. Reilly, A. Thomas, A. R. Gibson, C. Y. Luebehusen, M. D. Donohue Industrial \& Engineering Chemistry Research 2013; 52, 14456. 
(23) H. C. Struthers, F. M. Zehentbauer, E. Ono-Sorhue, J. Kiefer Industrial \& Engineering Chemistry Research 2011; 50, 12824.

(24) F. M. Zehentbauer, J. Kiefer Chimica Oggi/Chemistry Today 2012; 30, 54.

(25) F. Alsmeyer, H.-J. Koß, W. Marquardt Applied Spectroscopy 2004; 58, 975.

(26) K. Noack, B. Eskofier, J. Kiefer, C. Dilk, G. Bilow, M. Schirmer, R. Buchholz, A. Leipertz Analyst 2013; 138, 5639.

(27) J. Kiefer, K. Obert, A. Bösmann, T. Seeger, P. Wasserscheid, A. Leipertz ChemPhysChem 2008; 9, 1317.

(28) J. Kiefer, K. Obert, J. Fries, A. Bösmann, P. Wasserscheid, A. Leipertz Applied Spectroscopy 2009; 63, 1041.

(29) J. Kiefer, K. Obert, S. Himmler, P. S. Schulz, P. Wasserscheid, A. Leipertz ChemPhysChem 2008; 9, 2207.

(30) M. C. Weikl, F. Beyrau, J. Kiefer, T. Seeger, A. Leipertz Optics Letters 2006; 31, 1908.

(31) F. M. Zehentbauer, E. J. Bain, J. Kiefer Measurement Science and Technology 2012; $23,045602$. 